\title{
The Role of Interleukin-28b Gene Polymorphisms in Chinese Patients With Chronic Hepatitis C Treated With Pegylated Interferon and Ribavirin
}

\author{
Yin $\mathrm{Mi}^{1,2}$; Ying Tang Gao ${ }^{1}$; Xiao Lei Jiao ${ }^{1}$; Hua Guo ${ }^{1}$; Tong Liu $^{1}$; Li Jing ${ }^{1}$; Wen Xia Shi ${ }^{1}$; Zhi Du ${ }^{1, *}$ \\ ${ }_{1}^{1}$ Key Laboratory of Artificial Cell, Tianjin Third Central Hospital, Tianjin, China \\ ${ }^{2}$ Radiotherapy Department, First Affiliated Hospital, Zhengzhou University, Henan, China \\ ${ }^{*}$ Corresponding Author: Zhi Du, Key Laboratory of Artificial Cell, Tianjin Third Central Hospital, No. 83, Jintang Road, Hedong District, 300170, Tianjin, China. Tel: +86-2224315660, \\ Fax:+86-2224315132, E-mail: zhi-du@163.com
}

Received: March 6, 2014; Revised: May 4, 2014; Accepted: July 5, 2014

\begin{abstract}
Background:Interleukin-28B(IL28B) single nucleotide polymorphism(SNP) rs8099917 has been described to be associated with response to treatment with pegylated interferon and ribavirin (PEG-IFN/RBV) in patients with chronic hepatitis C from the North America, Europe, Asia countries like Japan and Taiwan. Whether this holds true for Chinese patients remains unknown.

Objectives: We aimed to study the effects of IL28B rs8099917 on antiviral therapy responses in Chinese patients with hepatitis C.

Patients and Methods: IL28B rs8099917 was genotyped in 263 patients with hepatitis C virus (HCV) infection and 244 healthy controls in Tianjin, China using TaqMan SNP genotyping method. The roles of rs8099917 and clinical characteristics in antiviral treatment were analyzed by logistic regression.

Results: Among 263 patients with chronic HCV infection, 223 had a TT genotype (84.8\%). Frequencies of TG/GG genotypes in patients with hepatitis $\mathrm{C}$ were significantly different from those of healthy controls (15.2\% vs. 9.0\%; $\mathrm{P}=0.033$ ). Patients with HCV infection had a higher $\mathrm{G}$ allele frequency than healthy controls ( $7.8 \%$ vs. $4.7 \% ; \mathrm{P}=0.044)$. Univariate analysis revealed no significant association between rs8099917 and sustained virological response (SVR) $(\mathrm{P}=0.612)$. However, it was found that HCV genotypes 2a/3a, age, prothrombin time (PT), albumin (ALB) and cholesterol (CHO) were associated with SVR. In multivariate analysis, only ALB was significantly an independent predictor of SVR $(\mathrm{OR}=1.223 ; 95 \% \mathrm{CI}: 1.046-1.430 ; \mathrm{P}=0.011)$.

Conclusions: In contrast with T, rs8099917 G is a susceptible allele to HCV in China. ALB can independently predict SVR. Rs8099917 may play a quiet role to predict treatment response of patients with hepatitis $C$ who received PEG -IFN/RBV therapy in China.
\end{abstract}

Keywords: China; Polymorphism; Hepatitis C; Interleukin-28B; Therapy

\section{Background}

HCV infected approximately 150 million people worldwide, resulting in death of up to 3.5 million people annually (1). Chronic HCV infection is a leading cause of liver cirrhosis and hepatocellular carcinoma (2). Current standard of care therapy for chronic HCV infection is PEG-IFN/ RBV; however only $40-50 \%$ of patients infected with HCV genotype 1 achieve SVR. Moreover, the treatment is associated with significant side effects, which is tolerated in few patients (3-6).

IL28B is also called IFN- $\lambda 3$ belonging to type III interferon family. The single nucleotide polymorphism (SNP) rs8099917 is located 8 kilobases upstream of the IL28B gene $(7,8)$. In recent years, three genome wide association studies identified the association between IL28B SNP rs8099917 and response to the treatment with PEG-IFN/ RBV among Caucasian and Japanese patients infected with HCV (7-9). Rauch et al. (7) performed a research on 1362 Caucasian patients treated with PEG-IFN/RBV. G was regarded as a risk allele owing to strong association between rs8099917 $\mathrm{G}$ allele and treatment failure in their study (OR = 5.19; 95\%CI: 2.90-9.30; $\left.\mathrm{P}=3.11 \times 10^{-8}\right)$. Suppiah et al. (8) documented that rs8099917 T allele could predict SVR $\left(\mathrm{OR}=1.98 ; 95 \% \mathrm{CI}: 1.57-2.52 ; \mathrm{P}=9.25 \times 10^{-9}\right)$. Furthermore, patients with TT genotype had a higher SVR rate compared to TG and GG genotypes. Afterwards several studies in cohorts of HCV genotype 1 patients in Japan, Taiwan, South Korea and Chile revealed that patients with TT genotype were more likely to obtain SVR than those carrying TG/GG genotypes (10-13). Paradoxically in the study undertaken by Chen et al. (14) in a Taiwanese cohort, GG genotype frequency of normal control males was 21.5 times more than that of male patients with HCV infection. GG could be considered as a protective genotype. In addition, Sinn et al. (15) studied Koreans with HCV genotype 1 infection and reported that there was no statistically difference in SVR rate between TT and TG/GG patients $(\mathrm{P}=0.19)$. Therefore, it can be seen that the association between IL28B rs8099917 and response to treatment is not definite in Asian patients with chronic hepatitis $C$. The prevalence rate of hepatitis C is 1-1.9\% in China (16) where seldom studies investigated the influence of rs8099917 on the treatment outcome of hepatitis $C$ patients with PEG-IFN/RBV. 
MiYetal.

\section{Objectives}

In the study, we detected the genotype and allele frequencies of IL28B rs8099917 in a Chinese cohort of patients with HCV infection and healthy people. Then we analyzed the impact of IL28B rs8099917 gene polymorphisms on patients' responses to therapy with PEG-IFN/ RBV with an attempt to offer certain evidences to clinical workers for effective individualized treatment and early assessment of patients.

\section{Patients and Methods}

\subsection{Patients}

We retrospectively enrolled 367 patients with chronic infection of HCV from 2005 to 2010 at Tianjin Third Central Hospital, Tianjin, China. In total, 158 patients were hospitalized and 209 were outpatients. Because of compliance problem, we actually genotyped IL28B and HCV in 263 and 176 patients, respectively. In total, 181 patients were treated for 48 weeks with peg-IFN- $\alpha 2 a(180 \mu \mathrm{g} /$ week; Shanghai Roche Co., LTD, Shanghai, China) subcutaneously and oral ribavirin (Shenyang Oasis Pharmaceutical Manufacturing Co., LTD, Shenyang, China) according to body weight $(<60$ $\mathrm{kg}, 800 \mathrm{mg} /$ day; $60-75 \mathrm{~kg}, 1000 \mathrm{mg} /$ day; and $>75 \mathrm{~kg}, 1200$ $\mathrm{mg}$ /day). The 181 patients' serum HCV RNA load results before treatment, after 12 weeks of treatment, at the end of treatment and at 24 weeks after completing therapy were documented in clinical records. IL28B rs8099917 was genotyped in 112 of 181 patients and HCV genotypes were detected in 89 of 112 patients (Figure 1). Chronic hepatitis $C$ was diagnosed in all patients included in the study according to the diagnostic criteria of hepatitis $\mathrm{C}$ (3). The exclusion criteria were as follows: human immunodeficiency virus infection, autoimmune hepatitis, a psychiatric condition and previous liver transplantation. Clinical data of each inpatient was obtained from their medical records by treating physicians. In addition, 244 healthy people who underwent health examination in the department of health of Tianjin Third Central Hospital were selected as healthy controls. This study was approved by the Ethical Committee of Tianjin Third Central Hospital, and informed consent was obtained from each patient before the study.

\subsection{Definition of Response}

For the analysis of response to standard of care PEG-FN/ RBV, SVR was defined as undetectable HCV RNA at the end of treatment and 24 weeks after the treatment completion. Relapse was defined as undetectable levels of HCV RNA at the end of treatment, while detectable HCV-RNA levels at 24 weeks after cessation of treatment. Patients whose HCV-RNA levels remained detectable at the end of treatment were considered to be nonresponders. Relapse and nonresponse composed treatment failure.

\subsection{HCV RNA Detection and HCV Genotyping}

Serum HCV RNA was measured using diagnostic kit for quantification of hepatitis C virus RNA (Shanghai Huake Bio-engineering Co., LTD, Shanghai, China) according to the manufacturer's protocol with a detection limit of 500 $\mathrm{IU} / \mathrm{mL}$. HCV genotyping was performed with the oligonucleotide DNA chip by the combined reverse transcriptionasymmetric polymerase chain reaction (PCR) and cohybridization methods (17).

\subsection{Genomic DNA Extracting}

Genomic DNA was obtained from peripheral blood white blood cells of patients and healthy controls with phenol-chloroform-isoamyl alcohol extraction method, and extraction results were detected in $1.5 \%$ agarose gel electrophoresis.

\subsection{IL28B rs8099917 Genotyping}

For 263 patients whose DNA was available, rs8099917 genotyping was performed by PCR amplification with ABI PRISM ® 7000 SDS (Applied Biosystems, Foster City, CA, USA). The PCR amplification mixture was $10 \mu \mathrm{L}$ in a total volume containing $1.5 \mu \mathrm{L}$ DNA template, $5 \mu \mathrm{L} 2 \times$ TaqMan Genotyping Master Mix, $0.25 \mu \mathrm{L} 40 \times$ TaqMan SNP Genotyping Assay Mix (rs8099917, C_11710096_10), and $3.25 \mu \mathrm{L}$ ultrapure water. The PCR reactions were performed in 96-well microplates with three negative control (NTC). Reaction conditions were: 50 cycles of thermal denaturation at $95{ }^{\circ} \mathrm{C}$ for 5 minutes, denaturation at $92^{\circ} \mathrm{C}$ for 20 seconds and annealing at $60^{\circ} \mathrm{C}$ for 1 minute. Two allelicspecific TaqMan probes were used to detect a specific SNP target. Allele discrimination was achieved by the detection of fluorescent signal and analysis of the distribution of the scatter plot chart using ABI PRISM @ 7000 SDS (Applied Biosystems, Foster City, CA, USA).

\subsection{Statistical Analysis}

The Hardy-Weinberg disequilibrium test was performed for SNP rs8099917 of patients group with chronic hepatitis $\mathrm{C}$ and healthy control group. The logarithmic transformation of the original values was performed before measuring serum HCV RNA levels. Comparisons between continuous variables in the two groups were performed using the Mann-Whitney U test or the Student T-test. Categorical data were compared using the $\chi^{2}$ test, correction for continuity or Fisher's exact test. Logistic regression analysis was performed to determine SVR predictors. The odds ratios (OR) and 95\% confidence intervals (95\% CI) were calculated. Data was analyzed by SPSS 17.0 statistical software package (SPSS Inc., Chicago, USA). All statistical analyses were based on the two-sided hypothesis tests, and Pvalues $<0.05$ were considered statistically significant.

\section{Results}

\subsection{Basic Demographic Characteristics of Patients With Chronic HCV Infection}

Basic demographic characteristics of patients are pre- 
MiYetal.

sented in Table 1 after consultation of clinical records and statistical disposal. There were 185 males (53.2\%). In total, HCV genotype was detected in 176 patients, with $132(75.0 \%)$ infected with HCV genotype 1 b, 43 (24.4\%) with HCV genotype $2 \mathrm{a}$ and $1(0.6 \%)$ with $3 \mathrm{a}$. The mean age was $56.7 \pm 12.8$ years. Among 158 inpatients, 96.8\% were Chinese Han, and the mean of prothrombin time (PT), albumin (ALB) and cholesterol (CHO) were $15.9 \pm$
2.62 seconds, $33.6 \pm 5.84 \mathrm{~g} / \mathrm{L}$ and $3.32 \pm 1.0^{6} \mathrm{mmol} / \mathrm{L}$, respectively. Liver cirrhosis was diagnosed in $88 \%$ of inpatients by clinical and radiographic data. Some medical records were not available due to retrospective nature of the study. Of 181 patients receiving PEG -IFN/RBV therapy, 63 obtained SVR, 73 were nonresponders and 45 relapsed. The overall SVR rate was $34.8 \%$ regardless of HCV genotype.

Figure 1. Overview of the Study Population

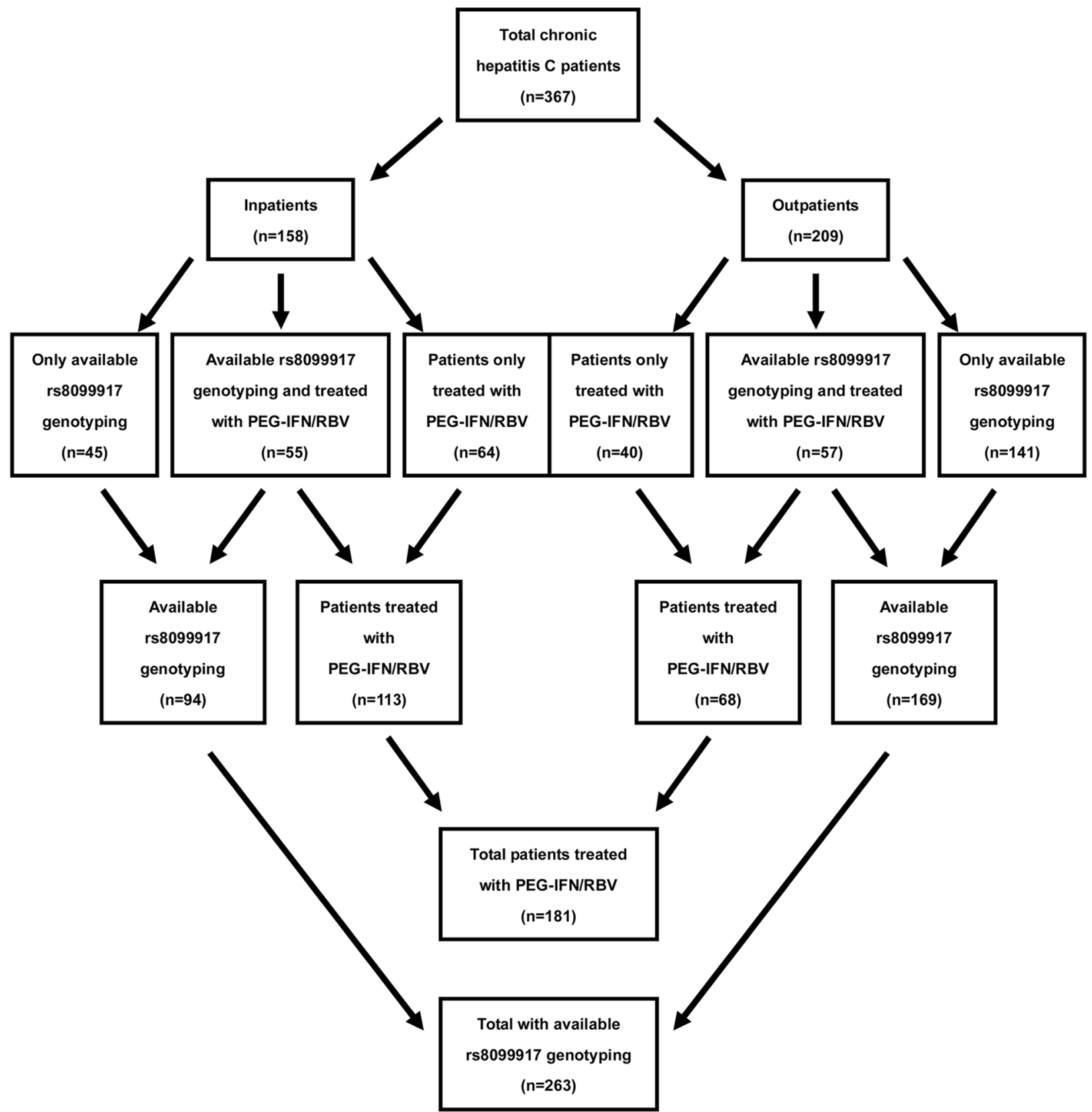

Rs8099917 genotyping is available in 112 patients treated with PEG-IFN/RBV. HCV genotype was detected in 89 patients. 
4.2. Comparison of rs8099917 Genotypes and Alleles Between Patients and Healthy Controls

Both genotypes distribution of patients and healthy controls were in Hardy-Weinberg equilibrium $\left(\chi^{2}=0.26\right.$; $\left.\chi^{2}=0.43\right)$ and both P values were more than 0.05 . Of 263 patients whose rs8099917 were genotyped, 223 (84.8\%) had the TT genotype, 39 (14.8\%) had the TG genotype, and $1(0.40 \%)$ carried GG genotype. The frequency of the rs8099917 T allele was 0.922. Among 244 healthy controls, the number of people who carried TT, TG and GG genotypes were $222(91.0 \%), 21(8.6 \%)$ and $1(0.4 \%)$, respectively. The $\mathrm{T}$ allele frequency was 0.953 (Table 2). TG/GG rate in patients with chronic hepatitis $\mathrm{C}$ was higher than that in healthy controls ( $15.2 \%$ vs. $9.0 \%$; OR $=1.810$; $95 \% \mathrm{CI}$ : 1.042-3.145; $\mathrm{P}=0.033$ ). Patients with chronic hepatitis $\mathrm{C}$ had a significantly higher frequency of $G$ allele compared with healthy controls ( $7.8 \%$ vs. $4.7 \%$; OR $=1.709 ; 95 \% \mathrm{CI}$ : 1.010-2.893; $\mathrm{P}=0.044)$. By comparison with $\mathrm{T}$, population with rs8099917 G allele is susceptible to HCV. Therefore, rs8099917 G was considered as a susceptible allele to HCV compared with T.

\subsection{Association of IL28B rs8099917 Gene Polymor- phisms and Treatment Outcome}

Regardless of HCV genotype, 112 patients with PEG-IFN/ RBV therapy whose rs8099917 were genotyped were included in this analysis. Among them, 97 had TT genotype and 15 carried TG/GG genotypes. The SVR rate in patients with TT genotype was higher than those with TG/GG genotypes; although, the difference was not statistically significant (40.2\% vs. $33.3 \%$; OR $=1.345 ; 95 \%$ CI: 0.427-4.237; $\mathrm{P}=0.612$, Table 3). The SVR rates for $\mathrm{T}$ and $\mathrm{G}$ alleles were $41.6 \%$ and $31.3 \%$, respectively. No significant difference was found likewise $(\mathrm{OR}=1.566$; 95\%CI: 0.526-4.666; $\mathrm{P}=0.417$, Table 3). Meanwhile, 89 of the 112 patients were genotyped for HCV. Of 72 patients with HCV genotype $1 \mathrm{~b}$ infection, no association was found between rs8099917 gene polymorphisms and treatment outcome $(\mathrm{P}=1.000$, Table 3). The association between rs 8099917 and treatment outcome was not analyzed solely among patients with HCV genotypes 2a/3a infection due to the small sample size.

\subsection{The Role of rs8099917 and Clinical Characteris- tics to Predict SVR}

The impacts of rs8099917 and clinical characteristics on SVR were evaluated by logistic regression (Table 4). It was found that there was no significant association between rs8099917 and SVR $(P=0.612)$ by univariate analysis. However, five variables associated with SVR were $\mathrm{HCV}$ genotypes $2 \mathrm{a} / 3 \mathrm{a}$ infection $(\mathrm{OR}=3.556 ; 95 \% \mathrm{CI}$ : $1.174-10.765 ; \mathrm{P}=0.044)$, age $(\mathrm{OR}=0.958 ; 95 \% \mathrm{CI}$ : 0.9290.987; $\mathrm{P}=0.005)$, PT ( $\mathrm{OR}=0.780 ; 95 \% \mathrm{CI}: 0.631-0.965 ; \mathrm{P}=$ $0.022), \operatorname{ALB}(\mathrm{OR}=1.188 ; 95 \% \mathrm{CI}: 1.086-1.300 ; \mathrm{P}=0.000)$ and $\mathrm{CHO}(\mathrm{OR}=2.078 ; 95 \% \mathrm{CI}: 1.224-3.531 ; \mathrm{P}=0.007)$. High SVR rate was more probable in patients with $\mathrm{HCV}$ genotypes 2a/3a infection, younger age, shorter PT, higher lever of ALB or CHO. Compared to 15 of 75 patients infected with HCV genotype $1 \mathrm{~b}, 8$ of 17 patients infected with HCV genotypes 2a/3a obtained SVR (SVR rate $20.0 \%$ vs. $47.1 \%$; $\mathrm{P}=0.044$ ). After excluding HCV genotype because of its many missing values, we assessed the relative contribution of important variables to treatment response in multivariate analysis and confirmed only ALB as an independent predictor of SVR (OR $=1.223$; $95 \%$ CI: 1.046 $1.430 ; \mathrm{P}=0.011$, Table 4).

Table 1. Basic Demographic Characteristics of Patients With Chronic HCV Infection ${ }^{a, b}$

\begin{tabular}{|c|c|c|}
\hline Clinical characteristics & No. & value \\
\hline Age, $y$, mean \pm SD & 320 & $56.7 \pm 12.8$ \\
\hline Male, n/No. (\%) & & $185 / 348(53.2)$ \\
\hline Han population, n/No. (\%) & & $153 / 158(96.8)$ \\
\hline HbsAg +, n/No. (\%) & & $14 / 117(12.0)$ \\
\hline GGT- II +, n/No. (\%) & & $23 / 125(18.4)$ \\
\hline Smoking history, n/No. (\%) & & $47 / 151(31.1)$ \\
\hline Drinking history, n/No. (\%) & & $39 / 153(25.5)$ \\
\hline Blood transfusion history, n/No. (\%) & & 28/158 (17.7) \\
\hline Liver cirrhosis & & $139 / 158(88.0)$ \\
\hline HCV 1b, n/No. (\%) & & $132 / 176(75.0)$ \\
\hline HCV-RNA > $400000 \mathrm{IU} / \mathrm{mL}, \mathrm{n} /$ No. (\%) & & $180 / 292(61.6)$ \\
\hline HCV-RNA, log IU/mL, median (IQR) & 292 & $5.97(5.18-6.56)$ \\
\hline ALT, U/L, median (IQR) & 158 & $55(31.8-96.5)$ \\
\hline AST, U/L, median (IQR) & 155 & $61(42-97)$ \\
\hline DBIL, umol/L, median (IQR) & 158 & $8.6(5.4-14.0)$ \\
\hline TBIL, umol/L, median (IQR) & 158 & $22.4(13.7-36.1)$ \\
\hline AFP, ng/mL, median (IQR) & 134 & $15.9(8.3-49.0)$ \\
\hline AFU, U/L, median (IQR) & 128 & $464.5(36.3-637.3)$ \\
\hline $\mathrm{PT}(\mathrm{s})$, mean $\pm \mathrm{SD}$ & 158 & $15.9 \pm 2.62$ \\
\hline PLT, $\times 10^{9} / \mathrm{L}$, mean \pm SD & 158 & $98.5 \pm 69.7$ \\
\hline $\mathbf{A L P}, \mathbf{U} / \mathbf{L}$, mean \pm SD & 156 & $91.0 \pm 41.3$ \\
\hline ALB, g/L, mean \pm SD & 157 & $33.6 \pm 5.84$ \\
\hline $\mathrm{CHO}, \mathrm{mmol} / \mathrm{L}$, mean $\pm \mathrm{SD}$ & 115 & $3.32 \pm 1.06$ \\
\hline TG, mmol/L, median (IQR) & 115 & $0.93(0.70-1.39)$ \\
\hline
\end{tabular}

a All data were obtained only from 158 inpatients except age, gender, HCV genotype and HCV virus load.

b Abbreviations: HbsAg, hepatitis B virus surface antigen; GGT- II, $\gamma$-glutamyl transferase isoenzymes $\Pi$; ALT, alanine aminotransferase;AST, aspartate aminotransferase; DBIL, direct bilirubin; TBIL, total bilirubin; AFP, alpha fetoprotein; AFU, $\alpha$-L-fucosidase; PT, prothrombin time; PLT, platelet; ALP, alkaline phosphatase; ALB, albumin; CHO, cholesterol; TG, triglyceride; IQR, Intra-quartile range (25th percentile-75th percentile); SD, standard deviation. 
MiYetal.

\begin{tabular}{|c|c|c|c|c|}
\hline & Healthy Controls, $N=244$ & Patients, $N=263$ & $\mathrm{OR}(95 \% \mathrm{CI})$ & P Value \\
\hline Genotype & & & & $0.033^{c}$ \\
\hline TT & $222(91.0)$ & $223(84.8)$ & 1 & \\
\hline TG & $21(8.6)$ & $39(14.8)$ & & \\
\hline GG & $1(0.4)$ & $1(0.4)$ & & \\
\hline TG/GG & $22(9.0)$ & $40(15.2)$ & $1.810(1.042-3.145)$ & \\
\hline Allele & & & & $0.044^{\mathrm{C}}$ \\
\hline $\mathrm{T}$ & $465(95.3)$ & $485(92.2)$ & 1 & \\
\hline G & $23(4.7)$ & $41(7.8)$ & $1.709(1.010-2.893)$ & \\
\hline Hardy-Weinberg, $\chi^{2}$ & 0.43 & 0.26 & & \\
\hline Hardy-Weinberg, P & $0.5<\mathrm{P}<0.75$ & $0.5<\mathrm{P}<0.75$ & & \\
\hline $\begin{array}{l}{ }^{\mathrm{a}} \text { Data are presented as } \mathrm{N} \\
\mathrm{b} \text { Abbreviations: OR, odd } \\
{ }^{c} \text { Pearson } \chi^{2} \text { test. }\end{array}$ & tio; CI, confidence interval. & & & \\
\hline
\end{tabular}

Table 3. Influence of IL-28B Rs8099917 Gene Polymorphisms on Treatment Outcome ${ }^{\text {a }}$

\begin{tabular}{|c|c|c|c|c|c|c|c|c|}
\hline & TT, No. (\%) & TG/GG, No. (\%) & OR $(95 \% C I)$ & Pvalue & T, No. (\%) & G, No. (\%) & OR $(95 \% \mathrm{CI})$ & P Value \\
\hline Total, $\mathbf{N}=112$ & & & & & & & & $0.417^{b}$ \\
\hline Treatment Failure & $58(59.8)$ & $10(66.7)$ & 1 & & $125(58.4)$ & $11(68.8)$ & 1 & \\
\hline SVR & $39(40.2)$ & $5(33.3)$ & $1.345(0.427-4.237)$ & $0.612^{b}$ & $89(41.6)$ & $5(31.3)$ & $1.566(0.526-4.666)$ & \\
\hline $\mathrm{HCV}_{1}{ }^{\mathrm{c}}, \mathrm{N}=\mathrm{72}^{\mathrm{c}}$ & & & & & & & & $1.000^{d}$ \\
\hline Treatment Failure & $49(79)$ & $8(80)$ & 1 & & $105(78.9)$ & $9(81.8)$ & 1 & \\
\hline SVR & $13(21)$ & $2(20)$ & $1.061(0.201-5.614)$ & $1.000^{d}$ & $28(21.1)$ & $2(18.2)$ & $1.200(0.245-5.872)$ & \\
\hline
\end{tabular}

\footnotetext{
a Abbreviations: SVR, sustained virological response; OR, odds ratio; CI, confidence interval.

$\mathrm{b}$ Pearson $\chi^{2}$ test

${ }^{c}$ Rs8099917 genotyping was not available in 3 of 75 patients infected with HCV genotype $1 \mathrm{~b}$

$\mathrm{d}$ Correction for continuity
} 
MiYetal.

\begin{tabular}{|c|c|c|c|c|c|}
\hline+ & & $0.816(0.261-2.556)$ & 0.727 & & \\
\hline HCV genotype & 92 & & & & \\
\hline $1 \mathrm{~b}$ & & 1 & & & \\
\hline $2 a / 3 a$ & & $3.556(1.174-10.765)$ & 0.044 & - & - \\
\hline HCV-RNA virus load & 160 & & & & \\
\hline HCV-RNA $>400000 I U / m L$ & & 1 & & & \\
\hline HCV-RNA $<400000 \mathrm{IU} / \mathrm{mL}$ & & $1.044(0.513-2.124)$ & 0.906 & & \\
\hline rs8099917 & 112 & & & & \\
\hline TG/GG & & 1 & & & \\
\hline TT & & $1.345(0.427-4.237)$ & 0.612 & & \\
\hline HCV-RNA, $\log \mathrm{IU} / \mathrm{mL}$ & 160 & $1.000(1.000-1.000)$ & 0.420 & & \\
\hline Age, $y$ & 171 & $0.958(0.929-0.987)$ & 0.005 & $0.997(0.942-1.056)$ & 0.923 \\
\hline ALT, U/L & 113 & $1.002(0.999-1.005)$ & 0.188 & & \\
\hline AST, U/L & 110 & $1.000(0.996-1.004)$ & 0.993 & & \\
\hline DBIL, umol/L & 113 & $0.959(0.911-1.010)$ & 0.115 & & \\
\hline TBIL, umol/L & 113 & $0.975(0.948-1.002)$ & 0.072 & & \\
\hline AFP, ng/mL & 95 & $1.000(0.999-1.001)$ & 0.567 & & \\
\hline AFU, U/L & 89 & $1.001(0.999-1.002)$ & 0.373 & & \\
\hline PT, $s$ & 113 & $0.780(0.631-0.965)$ & 0.022 & $0.967(0.643-1.456)$ & 0.874 \\
\hline PLT,$\times 10^{9} / \mathrm{L}$ & 113 & $1.004(0.998-1.010)$ & 0.162 & & \\
\hline ALP, U/L & 112 & $1.006(0.996-1.016)$ & 0.213 & & \\
\hline ALB, $\mathbf{g} / \mathbf{L}$ & 112 & $1.188(1.086-1.300)$ & 0.000 & $1.223(1.046-1.430)$ & 0.011 \\
\hline $\mathrm{CHO}, \mathrm{mmol} / \mathrm{L}$ & 82 & $2.078(1.224-3.531)$ & 0.007 & $1.424(0.753-2.694)$ & 0.277 \\
\hline TG, mmol/L & 82 & $1.300(0.907-1.863)$ & 0.153 & & \\
\hline
\end{tabular}

\footnotetext{
a Abbreviations: GGT- П , $\gamma$-glutamyl transferase isoenzymes $\Pi$; ALT, alanine aminotransferase; AST, aspartate aminotransferase; DBIL, direct bilirubin; TBIL, total bilirubin; AFP, alpha fetoprotein; AFU, $\alpha$-L-fucosidase; PT, prothrombin time; PLT, platelet; ALP, alkaline phosphatase; ALB, albumin; CHO, cholesterol; TG, triglyceride; OR, odds ratio; CI, confidence interval.

b Multivariate analysis was performed with factors significantly associated with SVR by univariate analysis except for HCV genotype because of its many missing values in multivariate analysis. Finally, multivariate analysis was performed in 82 patients.
}

\section{Discussion}

Not every patient with hepatitis $C$ infection receiving standard of care PEG-IFN/RBV could achieve SVR. Therefore, the treatment not only is costly but also has many side effects, which few patients could tolerate. If the predictors of treatment outcome be identified, the situation would be greatly improved. In addition to the viral and treatment factors, studies have found that IL28B gene polymorphism is closely related with the treatment response in hepatitis $C$ infection patients treated with PEG-IFN/RBV in the recent years. SNP rs8099917 and rs12979860 have a higher association with treatment response in comparison with other SNPs located in IL28B gene and are focused on in the recent years. Unfortunately, the association is diverse in different races and HCV genotypes in current studies. In this study, we detected the genotype and allele frequencies of IL28B rs8099917 in Chinese patients with HCV infection and healthy people and assessed the influence of IL28B rs8099917 gene polymorphisms on treatment responses in patients with chronic hepatitis $C$ treated with PEG-IFN/RBV.

So far, there have been few studies investigating the association between IL28B rs8099917 gene polymorphisms and treatment response to therapy with PEG-IFN/RBV in Chinese patients with chronic hepatitis C. In our study, frequencies of $\mathrm{T}$ allele in healthy people and patients chronically infected with HCV were $95.3 \%$ and $92.2 \%$, respectively. The frequencies of TT genotype were $91.0 \%$ and $84.8 \%$, respectively. Healthy people have higher frequen- 
MiYetal.

cies of $\mathrm{T}$ allele and TT genotype compared with patients with HCV infection ( $\mathrm{P}=0.044 ; \mathrm{P}=0.033)$. Therefore, TT genotype was presumed to be protective. Distributions of rs8099917 genotypes and alleles in our study are similar to those previously reported in other Asian countries and regions. As some studies reported, TT genotype frequencies of healthy people in Taiwan and Japan were 89.6\% and $83.7 \%$. While they were $89.6 \%, 86.2 \%$ and $70.4 \%$ for patients with HCV infection in Taiwan, Korea and Japan, respectively. Healthy people had a higher frequency of TT genotype than patients with chronic hepatitis $C$ in Japan. Dissimilarly, there was no obvious difference in the frequency of TT genotype between Taiwanese patients with hepatitis C and healthy people, and TT genotype frequency of Japanese patients with chronic hepatitis $C$ was lower than that of patients in other Asian countries and regions $(10,11,14,18)$. However, in European and Argentine patients infected with HCV, the frequency of TT genotype were $58.0 \%$ and $40.0 \%(7,19)$, respectively, which were both lower than those of Asian patients. This may to some extent explain why Asian patients with hepatitis $C$ receiving PEG-IFN/RBV treatment have a higher SVR rate than Caucasian ones (20).

In recent years, studies from different ethnic groups revealed that IL28B rs8099917 gene polymorphism was associated with treatment response to PEG -IFN/RBV in patients with HCV infection $(7,9,11,12,19)$. Suppiah et al. (8) evaluated 848 Caucasian patients with HCV genotype 1 infection and found that rs8099917 was related with $\operatorname{SVR}\left(\mathrm{OR}=1.98 ; 95 \% \mathrm{CI}: 1.57-2.52 ; \mathrm{P}=9.25 \times 10^{-9}\right)$. Among patients achieving SVR, 63\% had TT genotype while 37\% carried TG/GG genotypes. Tanaka et al. (9) investigated 142 patients infected with HCV genotype 1 within a Japanese population. The results demonstrated that rs8099917 was strongly associated with both SVR and null virological response $\left(\mathrm{P}=1.11 \times 10^{-27} ; \mathrm{P}=3.11 \times 10^{-15}\right)$. However, results of our research in Chinese patients with chronic $\mathrm{HCV}$ infection were not completely consistent with those previously reported. In our study though the SVR rate was higher in TT patients than TG/GG ones (40.2\% vs. $33.3 \%$ ), the difference was not statistically significant ( $\mathrm{P}$ $=0.612$ ). Rs8099917 gene polymorphism was not associated with SVR in patients with HCV genotype $1 \mathrm{~b}$ infection $(\mathrm{P}=1.000)$. Logistic regression showed that rs8099917 was not a predictor of SVR. Being similar to our results, Sinn et al. (15) reported that SVR rate did not differ significantly in HCV genotype 1 infection patients with TT genotype and TG/GG genotypes in Korea $(\mathrm{P}=0.19)$. Therefore, it is presumed that due to the high frequency of protective TT genotype and the low frequency of risk GG genotype, the IL28B gene polymorphism may play a quiet role in predicting treatment response of Chinese hepatitis C patients treated with PEG-IFN/RBV (15). On the other hand, the difference may be resulted from the small sample size in our study.

In addition, it was found by univariate analysis that $\mathrm{HCV}$ genotypes 2a/3a infection, younger age, shorter PT, higher level of ALB and CHO were associated with higher SVR rates. The findings that HCV genotypes 2a/3a, age and $\mathrm{CHO}$ are associated with SVR are in accordance with previous studies. Idrees et al. evaluated 400 patients with chronic hepatitis C (21). The result showed that patients with HCV genotypes 2a/3a infection or less than 40 years old had higher SVR rates $(P=0.022 ; P=0.005)$. In the study of Weich et al. (22), patients with HCV genotype 1 infection with a younger age or higher level of $\mathrm{CHO}$ were more likely to obtain SVR. Unlike previous studies which failed to consider the impact of PT and ALB on predicting treatment outcome, our study showed PT and ALB as predictors of SVR. These predictors of SVR may assist clinical physicians in early evaluating the progress of disease and patients prognosis.

SVR rates in our study were much lower than those previously reported. Standard of care PEG -IFN/RBV in this study leads to a SVR in $34.8 \%, 20.0 \%$ and $47.1 \%$ of total patients, patients infected with HCV genotype $1 \mathrm{~b}$ and HCV genotypes 2a/3a. A previous study showed that SVR was achieved in $42-52 \%$ of patients with HCV genotype 1 infection and $76-82 \%$ of those with HCV genotypes $2 / 3$ infection (23). The SVR rates in other Asian countries and regions (Japan, Taiwan, South Korea) were also higher than those in this study $(10,14,15)$. The following reasons may explain this problem. First of all, cirrhosis was a difficult to treat characteristic able to reduce SVR rate (24). Idrees et al. (21) found that none of 16 patients with cirrhosis achieved SVR in their study. In this study, patients with liver cirrhosis account for $88 \%$ of inpatients, which may lead to a low SVR rate. Although Ochi et al. (10) found higher SVR rates in Japanese and Taiwanese patients, patients in their study were in fibrosis stage not showing cirrhosis. Secondly, not all the outpatients selected in this study were treated initially. However, the specific number was not put on record. Retreatment of nonresponders usually produced low SVR rates ranging from $7 \%$ to $18 \%(24)$. Furthermore, small sample size may be another factor. Beside all the factors above, the fact that HCV genotype $1 \mathrm{~b}$ was predominant in this study (75\%) was associated with a decreased overall SVR to treatment.

In summary, this study analyzed the distribution feature of IL28B rs8099917 in a Chinese cohort and the effect of rs8099917 on treatment response to PEG-IFN/RBV in Chinese patients with HCV infection. Besides, supplemented the multiple ethnic studies of association between IL28B rs8099917 and treatment outcome of patients with chronic hepatitis C receiving PEG-IFN/RBV therapy and confirmed several predictors of treatment outcome to offer certain evidences to clinical practitioners for effective individualized treatment and early evaluation of patients. A major limitation of our study was the small number of patients with available rs8099917 genotyping and PEG-IFN/RBV therapy because of its retrospective nature. In future, there is a need for multicenter studies with larger sample size to identify the specific role of IL28B in antiviral treatment of patients with hepatitis C. 
MiYetal.

\begin{tabular}{|c|c|c|c|c|}
\hline Variable & No. & rs8099917, TT & rs8099917, TG/GG & P Value \\
\hline Gender, No. (\%) & 247 & & & $0.193^{b}$ \\
\hline Male & & $110(51.9)$ & $14(40.0)$ & \\
\hline Female & & $102(48.1)$ & $21(60.0)$ & \\
\hline HbsAg, No. (\%) & 71 & & & $1.000^{\mathrm{C}}$ \\
\hline+ & & $7(11.1)$ & $0(0)$ & \\
\hline- & & $56(88.9)$ & $8(100)$ & \\
\hline GGT- II , No. (\%) & 78 & & & $0.586^{c}$ \\
\hline+ & & $9(12.9)$ & $0(0)$ & \\
\hline- & & $61(87.1)$ & $8(100)$ & \\
\hline Blood transfusion history, No. (\%) & 94 & & & $0.724^{\mathrm{d}}$ \\
\hline Yes & & $17(20.2)$ & $1(10.0)$ & \\
\hline No & & $67(79.8)$ & $9(90.0)$ & \\
\hline HCV genotype, No. (\%) & 149 & & & $0.230^{d}$ \\
\hline $1 \mathrm{~b}$ & & $96(73.8)$ & $17(89.5)$ & \\
\hline $2 a / 3 a$ & & $34(26.2)$ & $2(10.5)$ & \\
\hline HCV-RNA virus load, No. (\%) & 211 & & & $0.395^{b}$ \\
\hline HCV-RNA > $400000 \mathrm{IU} / \mathrm{mL}$ & & $106(59.9)$ & $23(67.6)$ & \\
\hline HCV-RNA < $400000 I U / m L$ & & $71(40.1)$ & $11(32.4)$ & \\
\hline HCV-RNA, $\log$ IU/mL; median (IQR) & 211 & $5.95(5.19-6.61)$ & $5.95(5.42-6.35)$ & $0.610^{d}$ \\
\hline Age, y; mean \pm SD & 227 & $55.6(12.4)$ & $55.8(11.7)$ & $0.870^{\mathrm{e}}$ \\
\hline ALT, U/L; median (IQR) & 94 & $50(25.8-79.0)$ & $44(34.8-69.5)$ & $0.878^{\mathrm{e}}$ \\
\hline AST, U/L; median (IQR) & 92 & $57(34-95)$ & $60(28.0-85.5)$ & $0.655^{\mathrm{e}}$ \\
\hline DBIL, umol/L; median (IQR)) & 94 & $8.3(5.1-12.5)$ & $5.6(4.4-17.8)$ & $0.544^{\mathrm{e}}$ \\
\hline TBIL, umol/L; median (IQR) & 94 & $19.6(13.6-31.4)$ & $15.5(11.2-27.8)$ & $0.333^{\mathrm{e}}$ \\
\hline AFP, ng/mL; median (IQR) & 80 & $15.3(8.2-32.0)$ & $13.9(11.1-113.6)$ & $0.386^{\mathrm{e}}$ \\
\hline AFU, U/L; median (IQR) & 79 & $282(24-538)$ & $45.6(32-443)$ & $0.603^{e}$ \\
\hline PT, s; mean \pm SD & 94 & $15.8(2.45)$ & $14.8(1.23)$ & $0.199^{f}$ \\
\hline PLT, $\times 10^{9} / \mathrm{L} ;$ mean \pm SD & 94 & $100(63.7)$ & $95.7(55.5)$ & $0.835^{f}$ \\
\hline ALP, U/L; mean \pm SD & 92 & $85.5(37.4)$ & $93.9(36.2)$ & $0.523^{f}$ \\
\hline ALB, $g / L ;$ mean \pm SD & 93 & $33.8(6.09)$ & $37.1(5.17)$ & $0.109^{f}$ \\
\hline $\mathrm{CHO}, \mathrm{mmol} / \mathrm{L} ;$ mean $\pm \mathrm{SD}$ & 67 & $3.39(1.09)$ & $3.71(1.02)$ & $0.465^{f}$ \\
\hline $\mathrm{TG}, \mathrm{mmol} / \mathrm{L} ;$ mean $\pm \mathrm{SD}$ & 67 & $1.13(0.70)$ & $1.29(0.58)$ & $0.300^{f}$ \\
\hline
\end{tabular}

\footnotetext{
a Abbreviations: HbsAg, hepatitis B virus surface antigen; GGT-II, $\gamma$-glutamyl transferase isoenzymes II; ALT, alanine aminotransferase; AST, aspartate aminotransferase; DBIL, direct bilirubin; TBIL, total bilirubin; AFP, alpha fetoprotein; AFU, $\alpha$-L-fucosidase; PT, prothrombin time; PLT, platelet; ALP, alkaline phosphatase; ALB, albumin; CHO, cholesterol; TG, triglyceride; IQR, Intra-quartile range (25th percentile-75th percentile); SD, standard deviation.

$\mathrm{b}$ Pearson $\chi 2$ test.

${ }^{\mathrm{c}}$ Fisher's exact test.

$\mathrm{d}$ Correction for continuity.

$\mathrm{e}_{\text {Mann-Whitney U test. }}$

f Student t test.
} 
MiYet al.

Supplement 1. IL28B Rs8099917 Genotyping

A Delta Rn vs Cycle

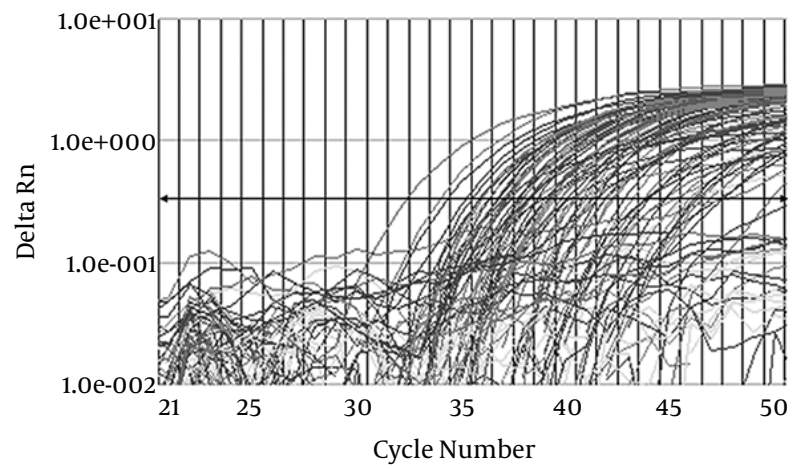

B

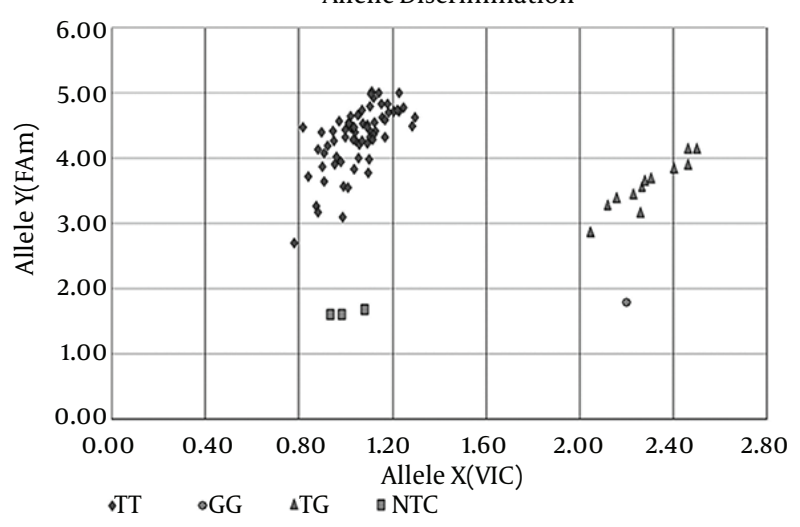

(A) PCR amplification curve of IL28B rs8099917. (B) Analysis graph of IL28B rs8099917 polymorphisms. Part of rs8099917 genotyping results are shown in this graph. Two allelic-specific TaqMan probes (VIC and FAM) were used to detect a specific SNP target. Rhombus, round and triangle indicate genotype TT, GG, and TG, respectively. Square represents negative control (NTC).

\section{Acknowledgements}

We would like to thank all of the study participants for their valuable contributions to this study.

\section{Authors' Contributions}

Ying Tang Gao and Yin Mi designed the study. Samples were collected by Hua Guo, Tong Liu, Li Jing and Wen Xia Shi. Yin Mi performed the experiments and did the statistical analysis. The manuscript was written by Yin Mi and revised by Ying Tang Gao, Xiao Lei Jiao and Zhi Du. All authors read and contributed to the manuscript.

\section{Funding/Support}

This research was supported by the Tianjin Third Central Hospital and Tianjin Key Laboratory of Artificial Cell, funded by grants from the Key Research Project of Tianjin Healthy Bureau (NO.11KG112).

\section{References}

1. Media Centre.. Hepatitis C.: WHO; 2012. Available from: http:// www.who.int/mediacentre/factsheets/fs164/en.

2. Seeff LB. Natural history of chronic hepatitis C. Hepatology. 2002;36(5 Suppl 1):S35-46.

3. Ghany MG, Strader DB, Thomas DL, Seeff LB, American Association for the Study of Liver D. Diagnosis, management, and treatment of hepatitis C: an update. Hepatology. 2009;49(4):1335-74.

4. Zeuzem S, Berg T, Moeller B, Hinrichsen H, Mauss S, Wedemeyer $\mathrm{H}$, et al. Expert opinion on the treatment of patients with chronic hepatitis C. J Viral Hepat. 2009;16(2):75-90.

5. Manns MP, McHutchison JG, Gordon SC, Rustgi VK, Shiffman $\mathrm{M}$, Reindollar $\mathrm{R}$, et al. Peginterferon alfa-2b plus ribavirin compared with interferon alfa-2b plus ribavirin for initial treatment of chronic hepatitis C: a randomised trial. Lancet. 2001;358(9286):958-65.

6. Fried MW, Shiffman ML, Reddy KR, Smith C, Marinos G, Goncales $\mathrm{FL}$, Jr, et al. Peginterferon alfa-2a plus ribavirin for chronic hepatitis C virus infection. N Engl J Med. 2002;347(13):975-82.

7. Rauch A, Kutalik Z, Descombes P, Cai T, Di Iulio J, Mueller T, et al. Genetic variation in IL28B is associated with chronic hepatitis C and treatment failure: a genome-wide association study. Gastroenterology. 2010;138(4):1338-45.

8. Suppiah V, Moldovan M, Ahlenstiel G, Berg T, Weltman M, Abate ML, et al. IL28B is associated with response to chronic hepatitis $\mathrm{C}$ interferon-alpha and ribavirin therapy. Nat Genet. 2009;41(10):1100-4

9. Tanaka Y, Nishida N, Sugiyama M, Kurosaki M, Matsuura K, Sakamoto N, et al. Genome-wide association of IL28B with response to pegylated interferon-alpha and ribavirin therapy for chronic hepatitis C. Nat Genet. 2009;41(10):1105-9.

10. Ochi H, Maekawa T, Abe H, Hayashida Y, Nakano R, Imamura M, et al. IL-28B predicts response to chronic hepatitis C therapy-finemapping and replication study in Asian populations.J Gen Virol. 2011;92(Pt 5):1071-81.

11. Lyoo K, Song MJ, Hur W, Choi JE, Hong SW, Kim CW, et al. Polymorphism near the IL28B gene in Korean hepatitis $C$ virus-infected patients treated with peg-interferon plus ribavirin. J Clin Virol. 2011;52(4):363-6.

12. Venegas M, Villanueva RA, Gonzalez K, Brahm J. IL28B polymorphisms associated with therapy response in Chilean chronic hepatitis C patients. World J Gastroenterol. 2011;17(31):3636-9.

13. Hsu CS, Hsu SJ, Chen HC, Tseng TC, Liu CH, Niu WF, et al. Association of IL28B gene variations with mathematical modeling of viral kinetics in chronic hepatitis $C$ patients with IFN plus ribavirin therapy. Proc Natl Acad Sci U S A. 2011;108(9):3719-24.

14. Chen JY, Lin CY, Wang CM, Lin YT, Kuo SN, Shiu CF, et al. IL28B genetic variations are associated with high sustained virological response (SVR) of interferon-alpha plus ribavirin therapy in Taiwanese chronic HCV infection. Genes Immun. 2011;12(4):300-9.

15. Sinn DH, Kim YJ, Lee ST, Gwak GY, Choi MS, Lee JH, et al. Association of a single nucleotide polymorphism near the interleukin$28 \mathrm{~B}$ gene with response to hepatitis $C$ therapy in Asian patients. $J$ Gastroenterol Hepatol. 2011;26(9):1374-9.

16. Sievert W, Altraif I, Razavi HA, Abdo A, Ahmed EA, Alomair A, et al. A systematic review of hepatitis C virus epidemiology in Asia, Australia and Egypt. Liver Int. 2011;31 Suppl 2:61-80.

17. Park JC, Kim JM, Kwon OJ, Lee KR, Chai YG, Oh HB. Development and clinical evaluation of a microarray for hepatitis $C$ virus genotyping. J Virol Methods. 2010;163(2):269-75.

18. Ito K, Higami K, Masaki N, Sugiyama M, Mukaide M, Saito H, et al. The rs8099917 polymorphism, when determined by a suitable genotyping method, is a better predictor for response to pegylated alpha interferon/ribavirin therapy in Japanese patients than other single nucleotide polymorphisms associated with interleukin-28B. J Clin Microbiol. 2011;49(5):1853-60.

19. Ridruejo E, Solano A, Marciano S, Galdame O, Adrover R, Cocozzella D, et al. Genetic variation in interleukin-28B predicts SVR in hepatitis $C$ genotype 1 Argentine patients treated with PEG IFN and ribavirin. Ann Hepatol. 2011;10(4):452-7.

20. Imazeki F, Yokosuka O, Omata M. Impact of IL-28B SNPs on con- 
trol of hepatitis $\mathrm{C}$ virus infection: a genome-wide association study. Expert Rev Anti Infect Ther. 2010;8(5):497-9.

21. Idrees M, Riazuddin S. A study of best positive predictors for sustained virologic response to interferon alpha plus ribavirin therapy in naive chronic hepatitis C patients. BMC Gastroenterol. 2009;9:5.

22. Weich V, Herrmann E, Chung TL, Sarrazin C, Hinrichsen H, Bug gisch P, et al. The determination of GGT is the most reliable pre- dictor of nonresponsiveness to interferon-alpha based therapy in HCV type-1 infection.J Gastroenterol. 2011;46(12):1427-36.

23. Hofmann WP, Zeuzem S. A new standard of care for the treatment of chronic HCV infection. Nat Rev Gastroenterol Hepatol. 2011;8(5):257-64.

24. Tsubota A, Fujise K, Namiki Y, Tada N. Peginterferon and ribavirin treatment for hepatitis C virus infection. World J Gastroenterol. 2011;17(4):419-32. 